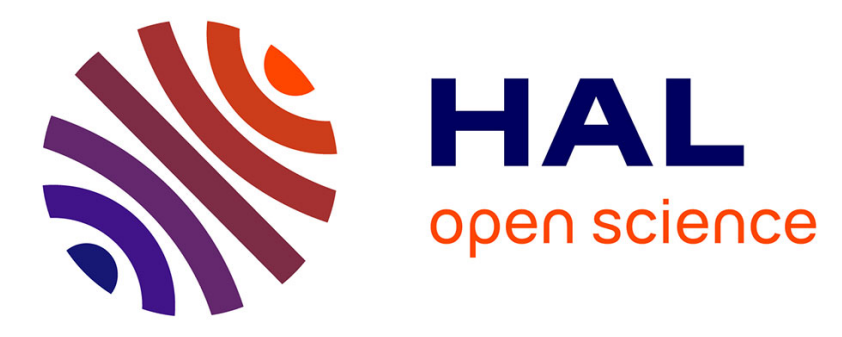

\title{
Novel Adaptive Controller for Effective Magnetic Measurement Under Arbitrary Magnetization
}

Anh Tuan Vo, Marylin Fassenet, Afef Kedous-Lebouc, François Blache, Cedric

Boudinet, Marie-Pierre Vaillant

\section{- To cite this version:}

Anh Tuan Vo, Marylin Fassenet, Afef Kedous-Lebouc, François Blache, Cedric Boudinet, et al.. Novel Adaptive Controller for Effective Magnetic Measurement Under Arbitrary Magnetization. IEEE Transactions on Industrial Electronics, 2020, 67 (11), pp.9841-9850. 10.1109/TIE.2019.2955422 . hal-02470966

\section{HAL Id: hal-02470966 https://hal.science/hal-02470966}

Submitted on 28 Nov 2020

HAL is a multi-disciplinary open access archive for the deposit and dissemination of scientific research documents, whether they are published or not. The documents may come from teaching and research institutions in France or abroad, or from public or private research centers.
L'archive ouverte pluridisciplinaire HAL, est destinée au dépôt et à la diffusion de documents scientifiques de niveau recherche, publiés ou non, émanant des établissements d'enseignement et de recherche français ou étrangers, des laboratoires publics ou privés. 


\section{Novel Adaptive Controller for Effective Magnetic Measurement Under Arbitrary Magnetization}

\begin{abstract}
In the context of the ever-expanding application of soft magnetic materials, the fully controlled magnetic measurement has, therefore, become essential. It ensures not only the accurate modeling of materials but also the rigorous quality control throughout the manufacturing process, as well as the explicit communication of magnetic data in academic studies or between suppliers and customers. Due to the non-linearity and hysteresis nature of electrical steels, automatic flux density controller is required for high standard measurements. We propose in this paper a novel steady-state digital control algorithm with two loops, one to regulate amplitude and the other to correct waveform of the flux density. Measurement results for various samples tested by Epstein frame and ring specimens under different waveforms, a wide range of frequency and high amplitudes of the flux density have proven the high adaptability, accuracy and convergence speed of this controller. Its principle is discussed in details, together with the employed measurement bench.
\end{abstract}

Index Terms-flux density controller, waveform controller, magnetic measurement, controlled magnetization, soft magnetic materials, electrical steels.

\section{INTRODUCTION}

$\mathbf{T}$ HE essential properties of magnetic materials such as power losses, permeability, and hysteresis loops are dependent on the flux density $B$ and the magnetic field strength $H$. Therefore, we generally measure the variation of $B$ within material samples with the externally applied $H$. The measurement is usually complicated due to the non-linearity introduced by the microstructure of materials [1], [2]. Also, the dynamic phenomena of magnetic materials, which are exhibited by the dependence of their properties on the rated change of $B$, can cause different testing results if the waveform of $B$ is not well controlled. Therefore, established standards such as IEC 60404 [3] have stated that sinusoidal flux density $B$ is required.

Thanks to breakthroughs of computer technology in recent decades, numerous hysteresis-based magnetic loss models have become more feasible and make it possible to predict iron losses in electromagnetic applications (electric rotating machines, transformers, etc.) with excellent accuracy [4]-[7]. However, these models usually require for their identification and validation more complex data (sometimes not easy to attain) comparing to classical analytical models. For instance, in [4], [5], authors imposed a high-amplitude triangular waveform on the flux density $B(t)$ to obtain major hysteresis loops with constant rated changes of $B$, whereas, $B(t)$ is traditionally a sinewave. Besides, material behavior is strongly dependent on the operating frequency of electromagnetic applications, which is increasing rapidly [8]-[11] due to the broad integration of switched-mode power supply. Therefore, there is a critical need for accurate and reliable magnetic mea-
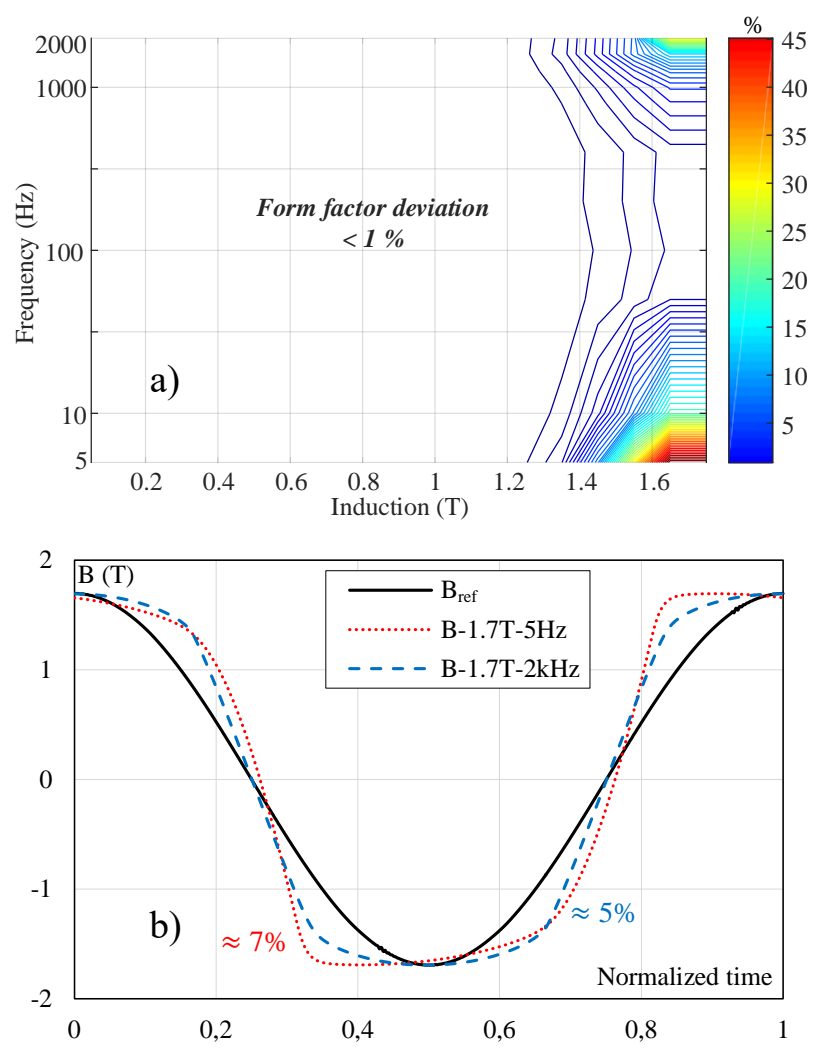

Fig. 1. Thin-gauge non-oriented Si-Fe NO20, sinewave flux density: (a) form factor deviation maps as a function of $B$ level $(0-1.7 \mathrm{~T})$ and frequency $(5-2000 \mathrm{~Hz}$ ), (b) flux density waveform with near saturation amplitude (1.7 T) at low and high frequency

surements, and so on, special controlled magnetizing devices.

Dealing with the crucial requirement of data acquisition for further numerical analysis, measurement systems naturally turn to digital. Computer-based systems with multifunctional high-speed sampling rate input/output devices are widely implemented, and digital feedback controllers [12][22] with their robustness have replaced the former analog circuits [23], [24] in most magnetic testing applications. The control of flux density waveform can be sophisticated in many cases because of the non-linearity of magnetic materials. For instance, as shown in Fig. 1a, without any flux controller, at critical conditions such as high amplitude and very low or high frequency, the deviation of the obtained flux density form factor exceeds $1 \%$ and thus violates the standard [3]. Typical distorted waveforms are illustrated in Fig. 1b. At low frequency, it is usually the parasitic resistance of the measurement bench which deforms the obtained $B(t)$; whereas the leakage flux of the magnetizing coil is the primary disturbance 
source at high frequency.

In [16] and [17], Zurek et al. reported an adaptive iterative feedback solution for $1 \mathrm{D}$ and 2D measurements, which was tested with diverse materials, frequency and amplitude levels of flux density. Without any use of parameter identification or particular adjustment depending on system configuration, this controller is supposed to be universal and has been applied in many reported studies, e.g. [10], [18]. However, the convergence time of their algorithm can be further improved. In [21], with the association of an analog circuit into a digital system, the convergence time is $95 \%$ reduced, but authors reported results for a quite narrow range of frequency $(2 \mathrm{~Hz}$ to $100 \mathrm{~Hz}$ ) and the analog circuit makes the system not feasible for general applications. In [12]-[14], model-based algorithms were presented, which may help to accelerate the measurement process. In contrast, some of their parameters vary following the magnetizing testers and must be experimentally identified. Thereby, these solutions are not considered universal.

In our study, we propose a novel digital feedback controller which can cooperate with various test systems and gives rapid, and accurate measurements without any extra parameters or practical choice of coefficients on material properties. The primary difference of this controller from the other published is the separation of the controlled variable $B(t)$ into two terms, the amplitude and the waveform. While the amplitude control loop is a classic proportional regulator, the waveform control loop is novel. It is triggered selectively to ensure the stability of the system and to acquire in a small number of iterations the required flux density $B(t)$. The principle of this controller is explained in the following section. At the end of this paper, two closed magnetizing yokes, including Epstein frame and ring specimens are used to test conventional and unconventional soft magnetic materials under both traditional and challenging arbitrary $\mathrm{AC}$ conditions.

\section{THE MEASUREMENT BENCH AND THE DIGITAL FEEDBACK FLUX CONTROLLER}

A test bench with a digital feedback structure and a highly adaptive control algorithm were developed to provide all measurements of soft magnetic materials with an entirely selfregulating mechanism.

\section{A. Description of the magnetic measurement bench}

The block diagram of the bench is presented in Fig. 2, and the technical details of principal components are found in Table I.

The most important component, which connects the entire system, is a computer-assisted LabVIEW program. The latter gathers feedback signals for the integrated controller and then generates a command signal to iteratively yield the measured flux density $B(t)$ to its reference $B_{\text {ref }}(t)$, within an acceptable amplitude and waveform error. Besides, all the data relating to the measured material, magnetizing yoke (tester), and sampling parameters as well are entered via the user interface of this program. The command signal $V_{1}(t)$ is directed to an arbitrary waveform generator that is in cascade with a voltage mode amplifier to power the tester. The primary side current
TABLE I

TECHNICAL DETAILS OF MEASUREMENT BENCH COMPONENTS

\begin{tabular}{|c|c|c|}
\hline Components & Reference & Technical details \\
\hline $\begin{array}{l}\text { Data acquisition } \\
\text { and generation card }\end{array}$ & $\begin{array}{l}\text { NI PXIe- } \\
6124\end{array}$ & $\begin{array}{l}4 \text { analog channels, sampling rate } 4 \\
\text { MS/s per channel, DAC resolution } \\
16 \text { bits, data transfer speed } 1.75 \mathrm{~GB} / \mathrm{s} \text {, } \\
\text { maximum output voltage used } 10 \mathrm{~V}, \\
\text { slew rate } 20 \mathrm{~V} / \mu \mathrm{s}\end{array}$ \\
\hline Current transducers & $\begin{array}{l}\text { LEM } \\
\text { IT } 60 \mathrm{~S} \\
\text { Ultrastab }\end{array}$ & $\begin{array}{l}\text { Closed loop Fluxgate IT type, } \\
\text { accuracy } 0.02725 \% \text {, measurement } \\
\text { range } 60 \text { A configurable, } \\
\text { Bandwidth- } 3 \mathrm{~dB} 500 \mathrm{kHz}\end{array}$ \\
\hline $\begin{array}{l}\text { Voltage conditioning } \\
\text { card }\end{array}$ & $\begin{array}{l}\text { TEGAM } \\
\text { 4040A }\end{array}$ & $\begin{array}{l}\text { Differential } 100 \mathrm{~V} \text { common mode } \\
\text { input, Bandwidth- } 3 \mathrm{~dB} 100 \mathrm{MHz} \text {, } \\
\text { attenuation factors } \div 1, \div 10, \\
\div 100 \text {, AC gain accuracy } 0.15 \mathrm{~dB}\end{array}$ \\
\hline $\begin{array}{l}\text { Voltage power } \\
\text { amplifier }\end{array}$ & $\begin{array}{l}\text { AE } \\
\text { TECHRON } \\
7796\end{array}$ & $\begin{array}{l}5000 \mathrm{~W} \text { peak continually, } 150 \mathrm{~V} \text { max, } \\
\text { Bandwith-3dB } 100 \mathrm{kHz} \text {, slew rate } \\
41 \mathrm{~V} / \mu \mathrm{s} \text {, phase response } \pm 8.3 \\
\text { degrees }(10 \mathrm{~Hz}-10 \mathrm{kHz})\end{array}$ \\
\hline
\end{tabular}

$I_{1}(t)$ and the secondary side voltage $V_{2}(t)$ of the tester are sensed by high bandwidth and linearity transducers. They are then converted to digital signals by data acquisition boards and finally sent to the LabVIEW program through very high-speed PXIe buses. From these signals, intermediate calculations are performed to find $B$ and $H$ based on Faraday and Ampère's laws (1).

$$
\left\{\begin{array}{rr}
B(t)= & \frac{1}{A_{m} N_{2}}\left\{\int_{0}^{t} V_{2}(\tau) d \tau-\frac{1}{T} \int_{0}^{T}\left(\int_{0}^{t} V_{2}(\tau) d \tau\right)\right\} \\
H(t)= & -\frac{N_{1} I_{1}(t)}{l}
\end{array}\right.
$$

where $l$ is the length of the mean magnetic path of the tester, $A_{m}$ and $A_{c 2}$ are the cross-section of the material sample and the secondary coil, $N_{1}$ and $N_{2}$ are respective the number of turns of the primary and the secondary side of the tester. These are must-known parameters for all indirect magnetic measurements. The term related to $H(t)$ in the $B(t)$ formula represents the stray magnetic flux caused by the difference of the material section and the measuring coil section.

At this stage, the input $(B, H)$ and the output $\left(V_{1}\right)$ of the controller have already been defined, and we propose then ideas how the controller is developed.

\section{B. Principle of the controller}

Firstly, we need a universal controller which is capable of regulating $B(t)$ in various waveforms, amplitudes, and magnetizing frequencies. A real-time controller is, therefore, challenging to satisfy this requirement because a complex waveform or a very high magnetizing frequency can lead to sudden changes of the input to which the system cannot respond and becomes unstable [25]. This is why a steady-state digital feedback controller with its robustness and straightforward approach is chosen, as in other recently published 


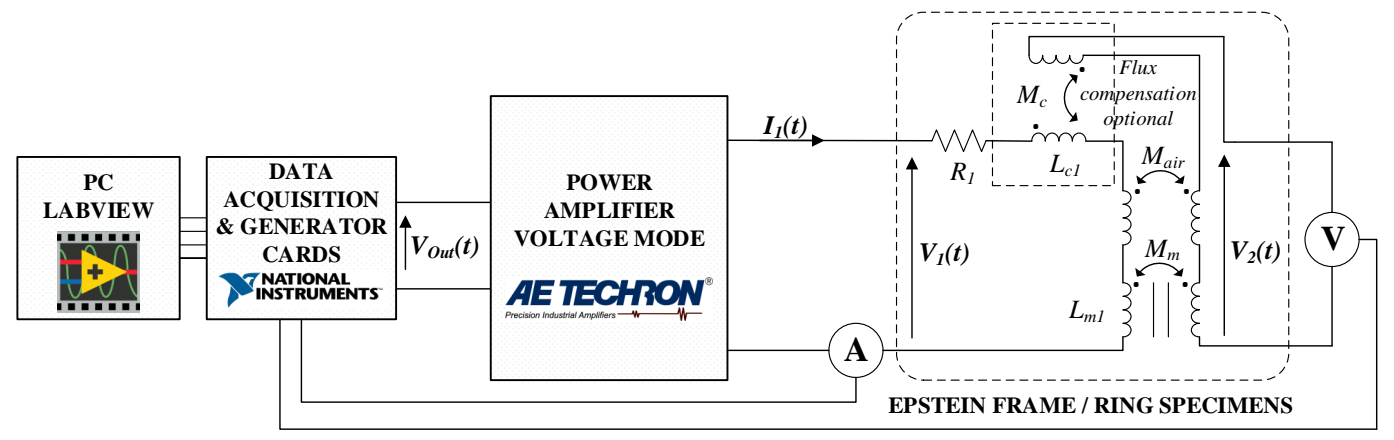

Fig. 2. Functional diagram of measurement bench with testers such as Epstein frame and ring cores

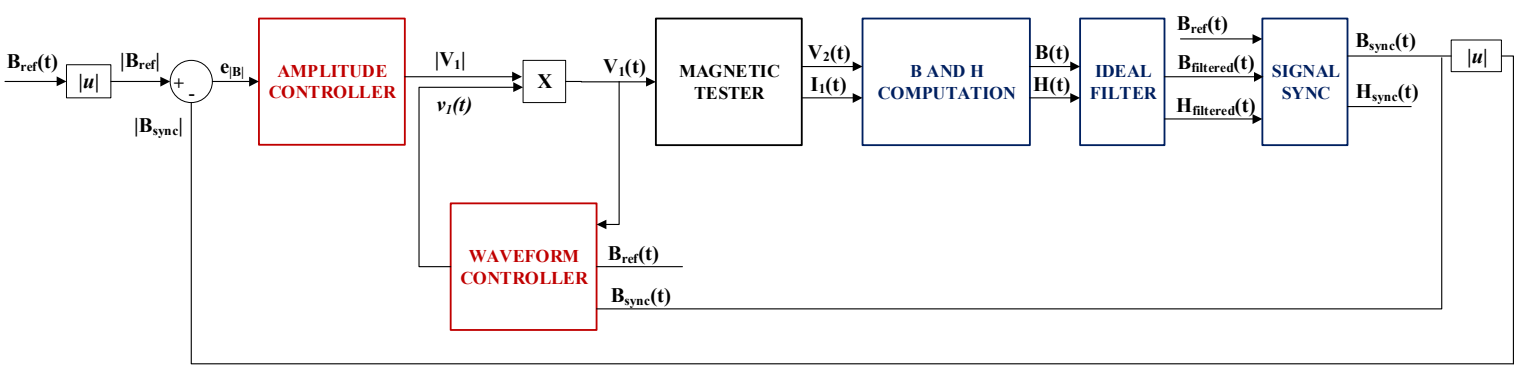

Fig. 3. Organization chart of the control algorithm

studies. It does not adjust the output in real time but only after the system has reached its steady-state. The waveform in an entire magnetizing period of $V_{1}(t)$ is corrected after each iteration, then several cycles of this voltage are sent to the tester so that the system response is stable at the end. Only the last stable cycles of the sampled signals are taken into account for the calculation of the next iteration. The signal averaging method, which sums the signal data and then generates an average cycle, is applied to reduce a part of measurement noise [26]. Consequently, the dynamics of each iteration do not have a critical effect on the controller stability.

Secondly, as the reference of magnetic flux in a magnetizing period $B_{r e f}(t)$ can be defined by its amplitude $\left|B_{r e f}\right|$ and normalized waveform $b_{r e f}(t)$ (unit amplitude) following the relationship in (2), we had an idea to realize a two-loop controller; an external loop yielding $|B|$ to $\left|B_{r e f}\right|$ and an internal one iteratively correcting $b(t)$ to obtain $b_{r e f}(t)$. The controlling signal $V_{1}(t)$ is also separated into $\left|V_{1}\right|$ and $v_{1}(t)$ respectively corresponding to the output of the internal and external control loop.

$$
\begin{gathered}
B_{r e f}(t)=\left|B_{r e f}\right| \cdot b_{r e f}(t) \\
B(t)=|B| \cdot b(t) ; V_{1}(t)=\left|V_{1}\right| \cdot v_{1}(t)
\end{gathered}
$$

During each control iteration, the primary side of tester circuit, as shown in Fig. 2, is modeled by formula (3).

$$
\begin{array}{r}
V_{1}{ }^{[i]}(t)=N_{1} A_{m} \frac{d B^{[i]}(t)}{d t}+R_{1} \frac{l}{N_{1}} H^{[i]}(t) \\
+N_{1}\left(A_{c 1}-A_{m}\right) \mu_{0} \frac{d H^{[i]}(t)}{d t}
\end{array}
$$

where $[i]$ and $[i+1]$ represent the $i^{t h}$ and $(i+1)^{t h}$ iteration respectively, $R_{1}$ is the total resistance of the tester primary side, $A_{c 1}$ is the cross section of the exciting coil.

If the magnetizing frequency is sufficiently high and the desired flux density is much lower than the saturation (valid in most cases as indicated in Fig. 1a), the last two terms of (3) are negligible. Consequently, $V_{1}{ }^{[i]}(t) \approx N_{1} A_{m} d B^{[i]}(t) / d t$, the waveform of $d B(t) / d t$ is imposed by the initial waveform of $V_{1}(t)$ and the internal control loop becomes useless. For example, we send a rectangular $V_{1}(t)$ to obtain a triangular $B(t)$. The idea of separating the two controlled variables is hence to reduce the calculation time and so the measurement time in these cases by automatically disabling the internal control loop. Moreover, in more critical cases as what we are addressing in this paper, the selective activation of this control loop also prevents the oscillation of $|B|$. The organization chart of the entire controller is presented in Fig. 3.

In the following sections, two control loops are described in detail, and it should be noticed that all time-dependent terms in equations are considered as the temporal variation of the variables over an entire period of magnetization.

1) Internal control loop: From the equivalent model in (3), we try to solve the inverse problem of how to iteratively modify the input $V_{1}(t)$ to obtain the required waveform $B_{\text {ref }}(t)$ of the flux density $B(t)$. The voltage and the magnetic field corresponding to $B_{r e f}(t)$ are interpreted respectively by $V_{1 r e f}(t)$ and $H_{r e f}(t)$. In case all parameters of the tester are well known, comprising $N_{1}, A_{m}, A_{c 1}, l$ and $R_{1}$, the two unknown terms are effectively $V_{1 r e f}(t)$ and $H_{r e f}(t)$. Supposing we have an accurate estimation of $H_{r e f}(t), V_{1 r e f}(t)$ can be 
directly derived by the following formula:

$$
\begin{array}{r}
V_{1 r e f}(t)=N_{1} A_{m} \frac{d B_{r e f}(t)}{d t}+R_{1} \frac{l}{N_{1}} H_{r e f}(t) \\
+N_{1}\left(A_{c 1}-A_{m}\right) \mu_{0} \frac{d H_{r e f}(t)}{d t}
\end{array}
$$

However, in the first iterations, such estimation is not possible. Moreover, what we have now, are $\left[\left|B^{[i]}\right| \cdot b(t), H^{[i]}(t)\right]$ with $\left|B^{[i]}\right| \neq\left|B_{r e f}\right|$ and $b(t) \neq b_{r e f}(t)$.

$V_{1 \text { ref }}(t)$ cannot be predicted to immediately obtain $B^{[i+1]}(t)=\left|B_{\text {ref }}\right| \cdot b_{\text {ref }}(t)$. The idea now is to correct $V_{1}^{[i]}(t)$ in a manner that in the next iteration $B^{[i+1]}(t)$ will have at least $\left|B^{[i+1]}\right|=\left|B^{[i]}\right|$ and $b^{[i+1]}(t) \approx b_{\text {ref }}(t)$. An estimation of $H^{[i+1]}(t)$ is required now, the simplest one is $H^{[i+1]}(t)=H^{[i]}(t)$, hence:

$$
\begin{array}{r}
V^{\prime[i+1]}(t)=N_{1} A_{m} \frac{d\left[\left|B^{[i]}\right| b_{r e f}(t)\right]}{d t}+R_{1} \frac{l}{N_{1}} H^{[i]}(t) \\
+N_{1}\left(A_{c 1}-A_{m}\right) \mu_{0} \frac{d H^{[i]}(t)}{d t}
\end{array}
$$

Subtracting both side of equation (3) and (5) yields a simpler expression for the correction of $V_{1}^{[i]}(t)$ after each iteration:

$$
V^{\prime[i+1]}(t)=V_{1}^{[i]}(t)+N_{1} A_{m} \frac{d}{d t}\left[\left|B^{[i]}\right| b_{r e f}(t)-B^{[i]}(t)\right]
$$

then

$$
\begin{array}{r}
V^{\prime[i+1]}(t)=V_{1}{ }^{[i]}(t)+N_{1} A_{m}\left|B^{[i]}\right| \frac{d}{d t}\left[b_{r e f}(t)-b^{[i]}(t)\right] \\
\text { with } V_{1}{ }^{[0]}(t)=N_{1} A_{m}\left|B_{\text {ref }}\right| \frac{d b_{r e f}(t)}{d t}
\end{array}
$$

The temporal derivative of $b(t)$ and $b_{r e f}(t)$ are calculated using the symmetric difference quotient block of LabVIEW. As $V_{1}^{\prime[i+1]}(t)$ is derived from $d b^{[i]}(t) / d t$, this feedback must be filtered to eliminate high-frequency measurement noise and be synchronized in phase with $b_{r e f}(t)$ (Fig. 4). The advantage of the steady-state digital feedback controller is its off-line nature, which allows the use of an ideal Fourier transformation instead of a real-time filter with phase lag for the filtering. Signals are analyzed in the frequency domain, all the harmonics beyond a pre-defined cut-off harmonic are then canceled. Before performing measurements, the cut-off harmonic, which depends significantly on the desired waveform, must be entered to the LabVIEW program with a value generally greater than 100 . The synchronization between $b^{[i]}(t)$ and $b_{r e f}(t)$ is managed by a particular numerical process which searches for the number of shifted elements of the array $b^{[i]}(t)$ minimizing the root mean square of residues between two signals.

$$
\min f_{k} \text { with } f_{k}=\sqrt{\sum_{j}\left[\operatorname{SHIFT}\left(b^{[i]}(j), k\right)-b_{r e f}(j)\right]^{2}}
$$

where $\operatorname{SHIFT}\left(b^{[i]}(j), k\right)$ means $b^{[i]}(t)$ is shifted $k$ elements.

As $V_{1}^{\prime[i+1]}(t)$ corresponds to the amplitude of $B(t)$ after the $i^{t h}$ iteration, $\left|B^{[i]}\right|$, whereas this value should converge towards its reference $\left|B_{\text {ref }}\right|$, an amplitude regulator is necessary. $V_{1}^{\prime[i+1]}(t)$ is normalized to have a unit amplitude waveform
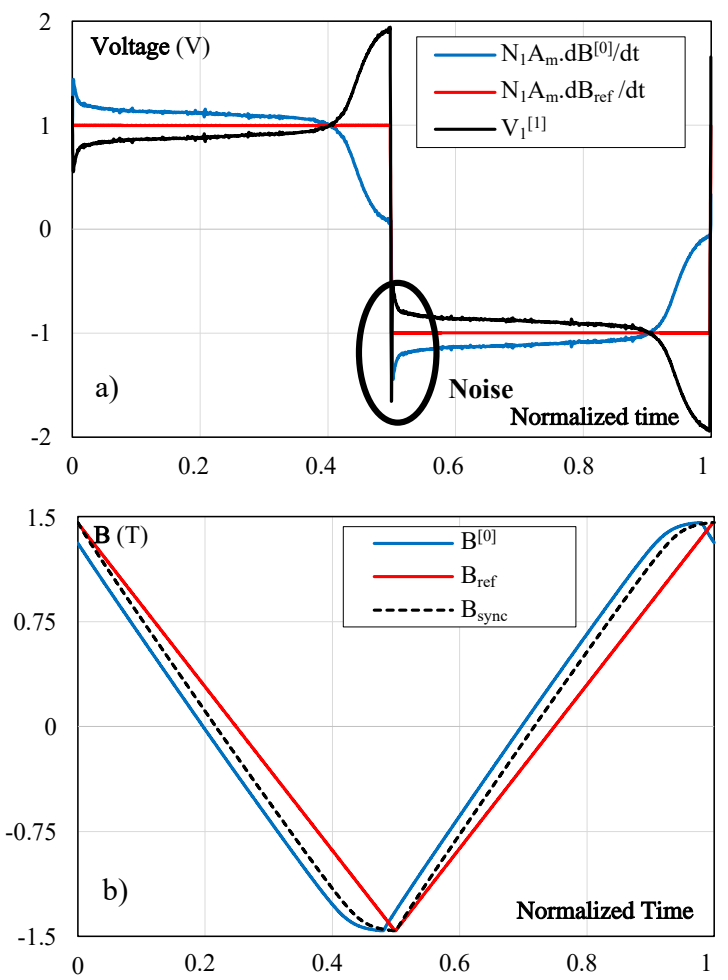

Fig. 4. Feedback signal processing: (a) high-frequency noise (black sudden peek) filtering, (b) signal sinchronization

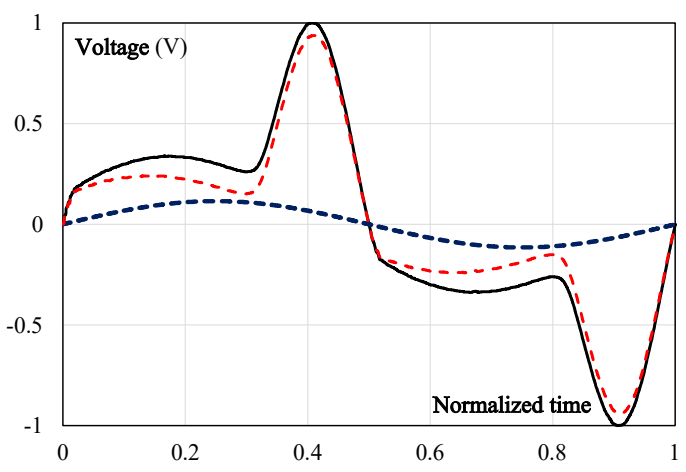

Fig. 5. Constituents of command signal $v_{1}(t)$ at very low frequency for sinusoidal signal, NO20: black full-line curve is $v_{1}(t)$, black dot-line curve is the first term of (3), red dash-line curve is the sum of the second and third term of (3)

$v_{1}^{[i+1]}(t)$ and next multiplied with the output of the external controller to obtain the control signal $V_{1}{ }^{[i+1]}(t)$.

$$
v_{1}^{\prime[i+1]}=\frac{V_{1}^{\prime[i+1]}}{\left|V_{1}^{[i+1]}\right|}
$$

2) External control loop: In case the last terms of (3) are negligible, the magnitude of two sides of (3) is given as:

$$
\begin{array}{r}
\left|V_{1}\right| \approx\left|V_{1}{ }^{[0]}\right|=N_{1} A_{m}\left|\frac{d B}{d t}\right|=N_{1} A_{m} K f|B| \\
\text { with } G=N_{1} A_{m} K f
\end{array}
$$

where $f$ is the magnetizing frequency and $K$ is a proportional constant between $|d B / d t|$ and $|B|$, for instance, in case of a sinewave we have $|d B / d t|=2 \pi f|B|$ and so on $K=2 \pi$. 


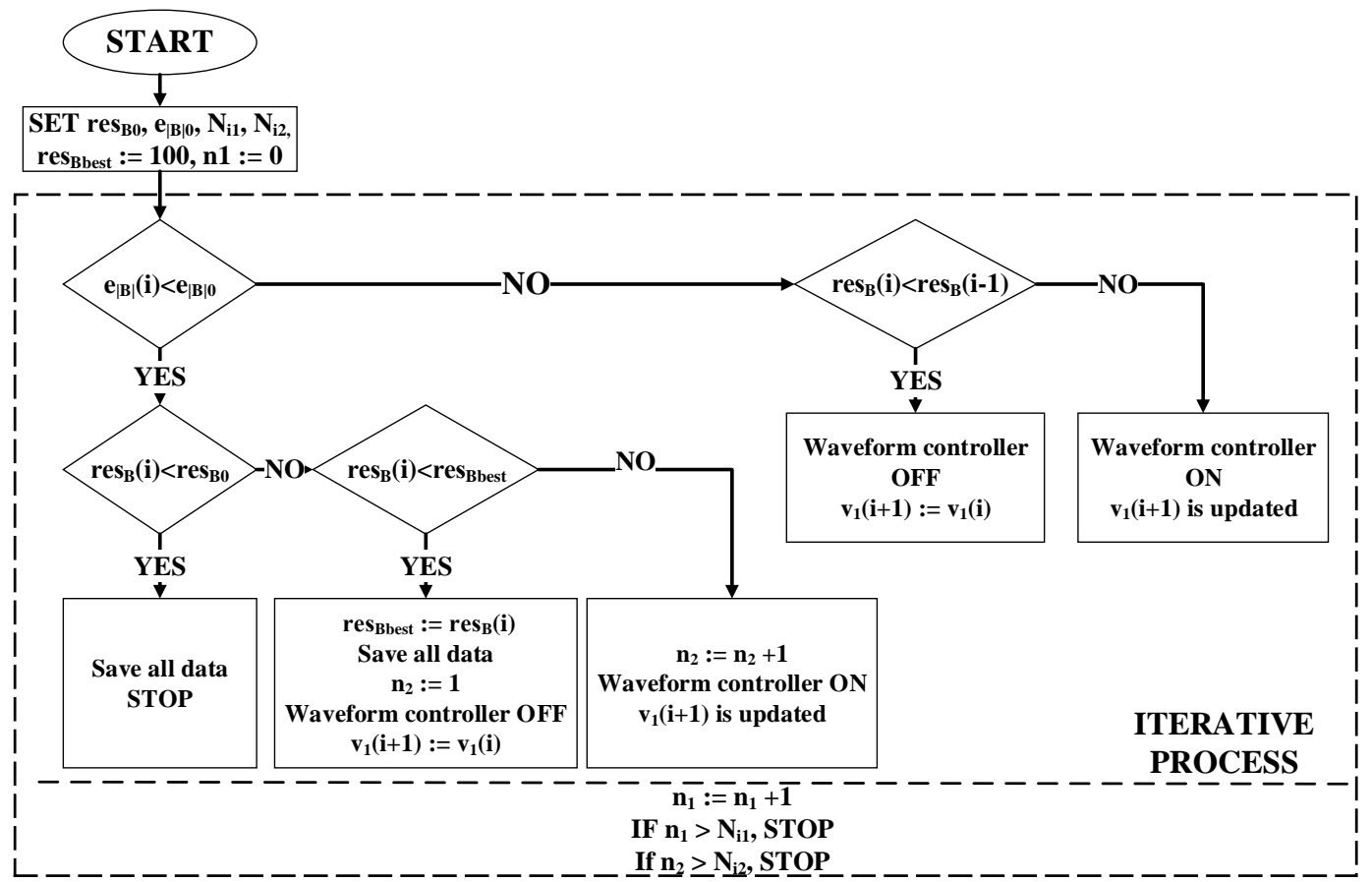

Fig. 6. Function diagram of the LabVIEW program

The proportional constant $G$ in (9) is simply determined by parameters of tester and waveform of $B(t)$ which must be known for all measurements with or without a controller. A straightforward digital proportional algorithm as following allows the flux density amplitude to achieve a sufficiently low steady-state error after two to three iterations:

$$
\begin{array}{r}
\left|V_{1}^{[i+1]}\right|=\left|V_{1}{ }^{[i]}\right|+K_{I}\left(\left|B_{\text {ref }}\right|-\left|B^{[i]}\right|\right) \\
\text { with }\left|V_{1}{ }^{[0]}\right|=K_{I}\left|B_{\text {ref }}\right|
\end{array}
$$

$K_{I}$ must be lower or equal to $G$ to limit the oscillation of $|B|$, in our study $K_{I}=G$.

As mentioned above, we are addressing measurements at extremely low or high frequency, and at a high amplitude of $B$. At low frequency, the $d B(t) / d t$ is small, and the first term of (3) becomes comparable to the second term. As the frequency increases, due to the limit of the power amplifier supply voltage, the number of material samples must be reduced. Thereby, the material section becomes much smaller than the tester coil section, and the third term is now non-negligible. In the above two cases, the waveform of $v_{1}(t)$ is highly distorted, as shown in Fig. 5. The system becomes exceptionally nonlinear and measurements are slowed down. Since $V_{1}{ }^{[i]}(t)$ is corrected to be $V_{1}^{\prime[i+1]}(t)$, (9) should be rewritten as:

$$
\left|V_{1}^{\prime}\right| \approx K_{M}\left|V_{1}^{[0]}\right|=K_{M} G|B|
$$

where the $K_{M}$ is employed for compensating for the tester non-linearity and accelerating the convergence of $B(t) . K_{M}$ is auto-updated after each iteration allowing the algorithm to converge at a better speed.

$$
K_{M}{ }^{[i]}=\frac{\int_{0}^{T}\left|v_{1}^{{ }^{[0]}}(t)\right| d t}{\int_{0}^{T}\left|v_{1}^{[i]}(t)\right| d t} \text { and } K_{M}^{[0]}=1
$$

Finally, the amplitude control loop has the following form:

$$
\begin{array}{r}
\left|V_{1}^{[i+1]}\right|=\left|V_{1}^{\left.{ }^{[i+1]}\right]}\right|+K_{M}{ }^{[i+1]} K_{I}\left(\left|B_{\text {ref }}\right|-\left|B^{[i]}\right|\right) \\
\text { with } K_{I}=G
\end{array}
$$

From this stage, the control voltage sent to the tester after each iteration is $V_{1}{ }^{[i+1]}(t)=\left|V_{1}{ }^{[i+1]}\right| \cdot v_{1}^{\prime[+1]}(t)$.

\section{Implementation of the controller in LabVIEW}

The controller is implemented in the LabVIEW program according to the function diagram shown in Fig. 6. Two main points that should be noted is the manner the two control loops cooperate and the stopping criteria of measurements.

The external controller is always on to continually regulate the amplitude of $B(t)$, whereas the internal one is only triggered in the two following cases based on the value of the waveform indicator $\mathrm{res}_{B}$ to avoid the oscillation of amplitude:

1) the amplitude of $B(t)$ is increasing, but the $\operatorname{res}_{B}$ value increases too, which means $b(t)$ is becoming more distorted, the internal controller is necessary to improve $\operatorname{res}_{B}$;

2) at the end of each iteration that the amplitude error $e_{|B|}$ is satisfied, the $r e s_{B}$ value is recorded and compared with the previously recorded values. If the actual $\mathrm{res}_{B}$ is not better than the previous best one, then the internal controller is activated to search for a better $v_{1}(t)$. 
The amplitude error of $B(t)$ is evaluated as follows:

$$
e_{|B|}=\frac{\left|B_{r e f}\right|-|B|}{\left|B_{\text {ref }}\right|} \cdot 100 \%
$$

The distortion of $B(t)$ is evaluated by the waveform indicator by (15). The traditional form factor is not employed because of its ambiguity for non-sinusoidal waveforms.

$$
\operatorname{res}_{B}=\sqrt{\sum_{j=1}^{N} \frac{\left(b^{[j]}-b_{r e f}{ }^{[j]}\right)^{2}}{N}} \cdot 100 \%
$$

where $\mathrm{N}$ is the number of samples per period of $B(t), j$ is the element position in the array.

Before executing the program, the desired value of amplitude error $e_{|B| 0}$ and waveform indicator $\operatorname{res}_{B 0}$ must be entered, the lower these desired values are, the more convergence time the system requires. Besides, we also need to define the maximum number of iterations in total $N_{i 1}$ and the maximum number of iterations at which we have $e_{|B|}<e_{|B| 0}$ before stopping the measurement $N_{i 2}$. These latter parameters are useful in case the program encounters the convergence problem, and measurement lasts too long. The measurement program stops in the four following cases:

1) the measurement is successfully performed, which means the amplitude error $e_{|B|}$ and the waveform indicator res $_{B}$ are both lower than their required values;

2) the maximum number of iterations is reached, but $b(t)$ does not meet its required waveform, the system automatically stops to avoid thermal overload;

3) the current or the voltage reaches their limit, and the program is automatically urgent shut down;

4) the user intends to shut down the system because it might seem never converge or unsecured problems occur or simply because he does not want to continue the measurement;

As soon as the measurement is finished, all feedback signals and derived $B(t)$ and $H(t)$ are saved. The user can display the hysteresis loop, signal waveforms, and also relevant information such as specific power losses, permeability, magnetic remanence, and coercivity.

\section{Results}

The flux controller was tested with two magnetizing circuits, including Epstein frame and ring cores. For various measurement conditions, we found results of high accuracy and short convergence time that proved the great flexibility of the controller. As illustrated in Fig. 7, a measurement with thin-gauge non-oriented $\mathrm{SiFe} \mathrm{NO} 20$ samples was carried out to demonstrate the iterative adjustment of the control waveform $v_{1}(t)$ and the respective evolution of $B(t)$. After six iterations, $B(t)$ converges to $B_{r e f}(t)$, firstly the amplitude and then the waveform. The voltage $v_{1}(t)$ is distorted rapidly as the flux is closer to its saturation value. By comparing $v_{1}{ }^{[5]}(t)$ and $H^{[5]}(t)$, we can see that the waveform of $v_{1}(t)$ was considerably modified under the influence of very high magnetic field (maximum value nearly $10000 \mathrm{~A} / \mathrm{m}$ ).

Performance analysis of the controller is conducted using three measurements in the critical domain of Fig. 1a. The
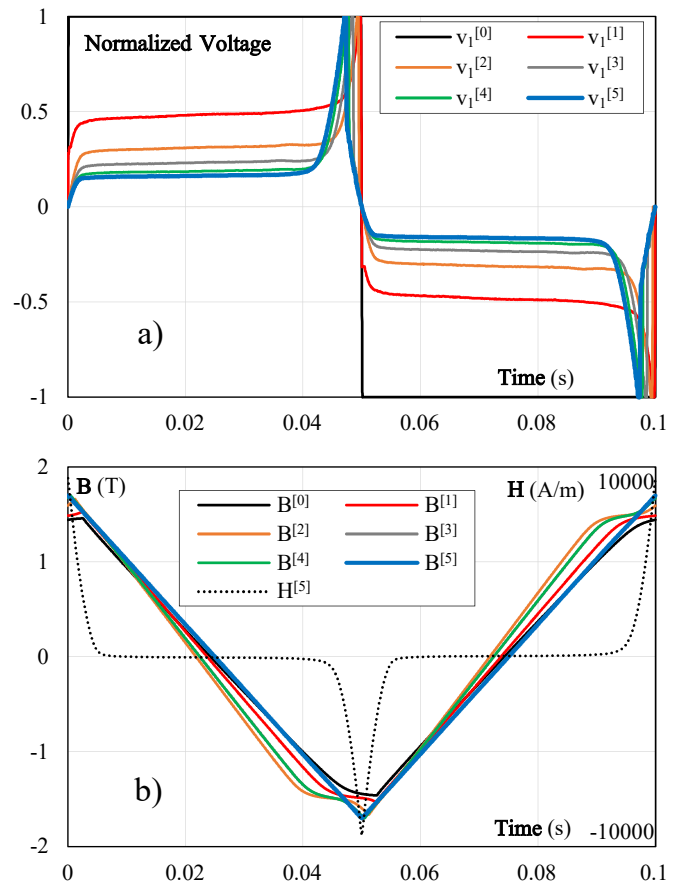

Fig. 7. NO20, triangular $B(t)$ at $10 \mathrm{~Hz}, 1.7 \mathrm{~T}$ : (a) the iterative change of $v_{1}(t)$, (b) $H(t)$ at the last iteration \& the evolution of $B(t)$
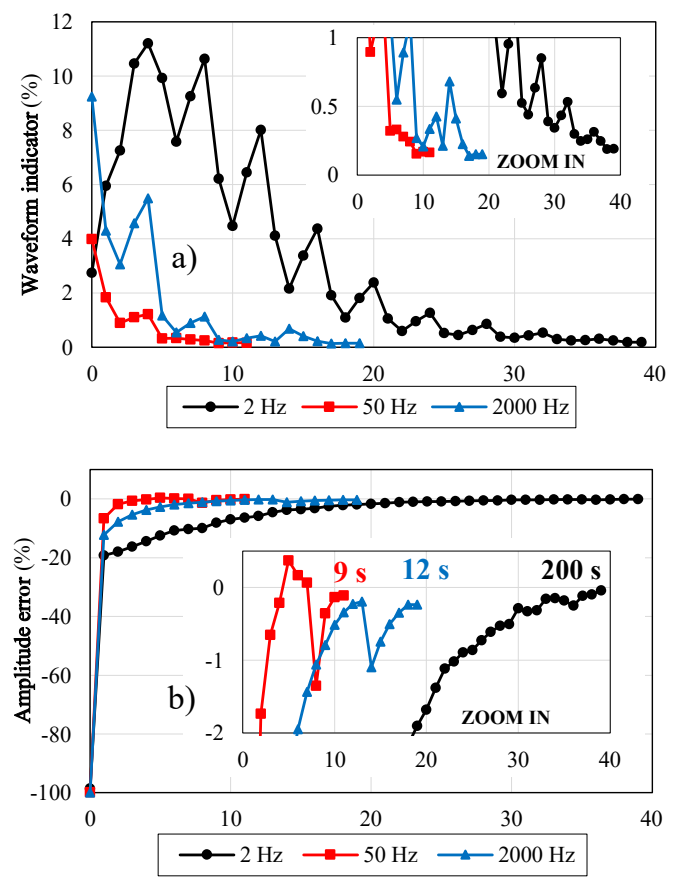

Fig. 8. NO20, sinusoidal $B(t)$ at $1.7 \mathrm{~T}$ : the evolution of the waveform indicator (a) and the amplitude error (b) in function of iteration

evolution of amplitude error and waveform indicator of $B(t)$ as functions of the iteration number are represented in Fig. $8 \mathrm{a}$ and Fig. 8b respectively. It can be observed that, in the end, these criteria are below their desired value of $0.2 \%$. The waveform quality is ensured much higher than that required by the IEC standard [3]. At the regular frequency $50 \mathrm{~Hz}$, the controller manages to stop correctly after about 10 iterations, 


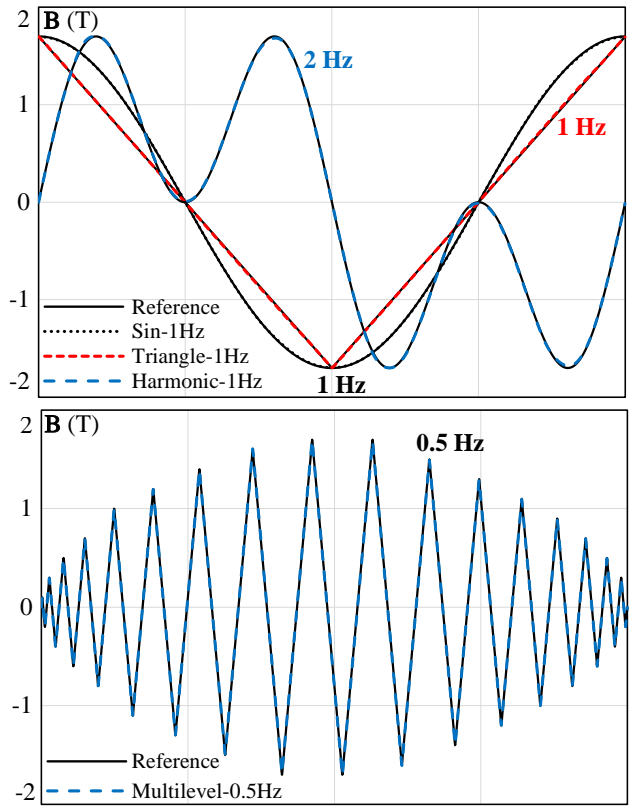

a)

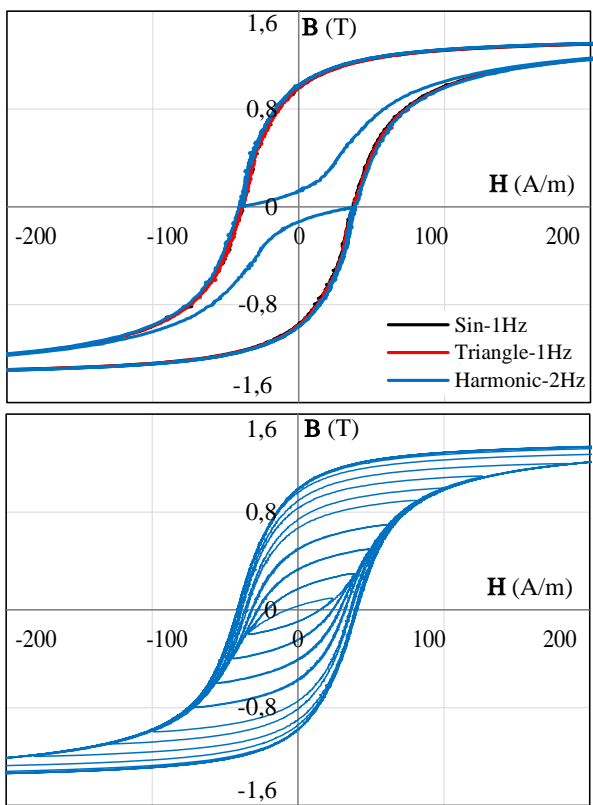

Fig. 9. NO20, quasi-static measurements $1.7 \mathrm{~T}$ : (a) triangular $1 \mathrm{~Hz}, \sin 1 \mathrm{~Hz}$ and multi-harmonic $2 \mathrm{~Hz}$, (b) user-defined waveform at $0.5 \mathrm{~Hz}$

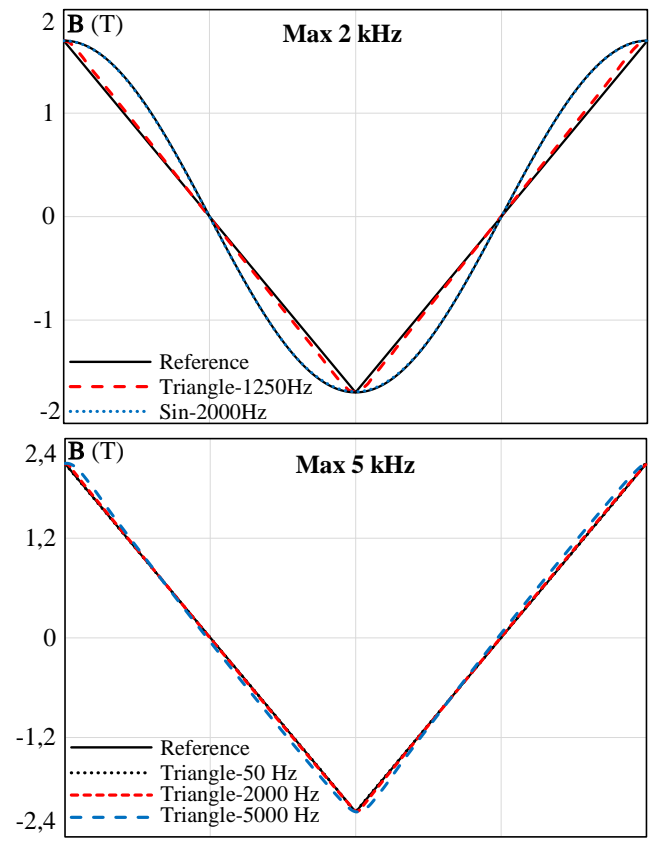

a)

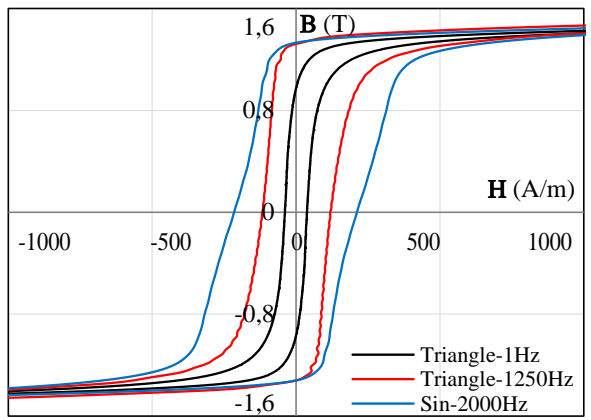

b)

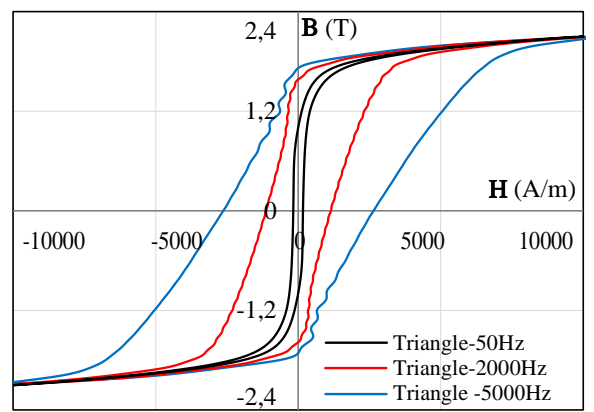

Fig. 10. Dynamic measurements: (a) NO20 1.7 T, Epstein frame, triangular $1.25 \mathrm{kHz}, \sin 2 \mathrm{kHz}$, (b) Co-Fe $2.1 \mathrm{~T}$, ring cores, triangular up to $5 \mathrm{kHz}$

whereas it takes respectively about 20 and 40 iterations at very high frequency $(2 \mathrm{kHz})$ and very low frequency $(2 \mathrm{~Hz})$ due to the rival influence of the steel alloy non-linearity. As indicated in the principle of the controller section, the more non-linear the system, the longer the measurement duration.

The average duration for each iteration at $2 \mathrm{~Hz}, 50 \mathrm{~Hz}$, and $2 \mathrm{kHz}$ are respectively 0.9 second, 0.6 seconds and 0.5 seconds. During each iteration, the controller must wait for the magnetizing operation, then process the feedback and send the control signal to the generator. These tasks vary in different ways, depending on the frequency and properties of materials. Supposing the number of cycles sent to the tester per iteration is fixed for all frequencies, the lower the magnetizing frequency we need, the longer its period is, and hence the magnetizing operation lasts longer at low frequency than at high frequency.

The controller is versatile, and its performance is highly dependent on user demand. As found in Fig. 8, the measurement can be accelerated by decreasing the quality criteria. For example, considering the desired waveform indicator of $0.5 \%$, we can reduce nearly half of iterations in every measurement. In Fig. 9, NO20 alloy is measured in quasi-static conditions with very low frequency $(0.5$ to $2 \mathrm{~Hz})$ and high amplitude of induction $(1.7 \mathrm{~T})$. On the left side, we can see that the 

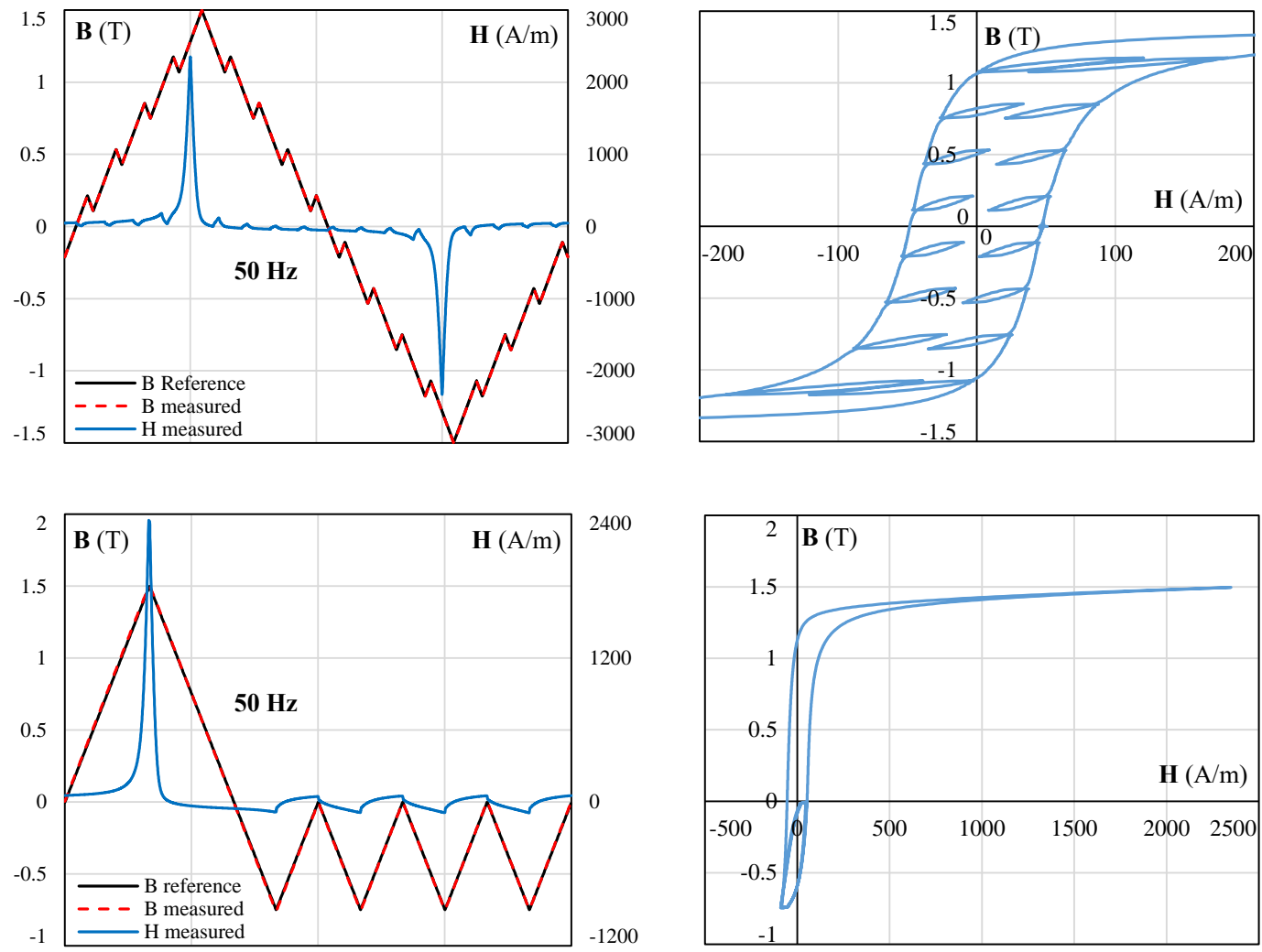

Fig. 11. Complex measurements: (a) NO20 1.5 T, Epstein frame, PWM $50 \mathrm{~Hz}$, (b) NO20 1.5 T, Epstein frame, asymmetrical triangular $50 \mathrm{~Hz}$

TABLE II

SUMMARY OF CONTROLLER PERFORMANCE

\begin{tabular}{|c|c|c|c|c|c|}
\hline \multirow{2}{*}{ Tested materials } & \multicolumn{2}{|c|}{ Frequency range $(\mathrm{Hz})$} & \multirow{2}{*}{$\begin{array}{l}\text { Induction amplitude } \\
\text { / Saturation }(\mathrm{T})\end{array}$} & \multirow{2}{*}{ Tested waveform } & \multirow{2}{*}{ Convergence time } \\
\hline & Epstein frame & Ring samples & & & \\
\hline $\begin{array}{l}\text { Grain oriented } \mathrm{SiFe} \text {, classical Non- } \\
\text { oriented } \mathrm{SiFe}\end{array}$ & & & $1.9 / 2.0$ & \multirow{6}{*}{$\begin{array}{l}\text { Sinusoidal, } \\
\text { triangular, } \\
\text { trapezoidal, } \\
\text { multi-harmonic, } \\
\text { PWM, } \\
\text { complex } \\
\text { user-defined }\end{array}$} & \multirow{5}{*}{$\begin{array}{l}\cdot 10 \mathrm{~s} \text { for typical } \\
\text { measurements } \\
\cdot<5 \text { minutes for } \\
\text { quasi-static } \\
\text { measurements }\end{array}$} \\
\hline Thin-gauge Grain Non-oriented SiFe & 12000 & 1 5000 & $1.75 / 2.0$ & & \\
\hline Si-enriched Grain Non-oriented $\mathrm{SiFe}$ & $1-2000$ & $1-5000$ & $1.7 / 1.8$ & & \\
\hline $\mathrm{NiFe}$ & & & $1.55 / 1.6$ & & \\
\hline Amorphous & $\begin{array}{c}\text { mini-frame } \\
1-5000\end{array}$ & $1-10000$ & $1.5 / 1.56$ & & \\
\hline Nanocrystalline & - & $1-10000$ & $1.1 / 1.2$ & & \\
\hline
\end{tabular}

obtained induction signals superimpose their references. Their hysteresis cycles (maximum value reaches $8000 \mathrm{~A} / \mathrm{m}$ ) are not presented in full scale to precise the shape of minor loops. The induction waveform, such as triangular and multi-harmonics, are widely used for the hysteresis modeling of electrical steels and measurements are required to have excellent accuracy. The last one with complex shape and many turning points at different levels, which can be applied for the validation of hysteresis models, is generally a challenge for any flux controller. However, we obtained high-quality results with the novel controller.

The results of dynamic measurements are also presented in Fig. 10, NO20 is used for the first two figures and a CoFe alloy for the others. With NO20, Epstein samples are employed for the measurement, which allows the frequency to reach 1250 $\mathrm{Hz}$ and $2000 \mathrm{~Hz}$ respectively in case of triangular and sinusoidal waveforms. Although the frequency increment inflates the shape of hysteresis cycles, measurements are performed accurately. Then, CoFe ring specimens are measured with a frequency of up to $5 \mathrm{kHz}$ at the $2.1 \mathrm{~T}$ induction level. This alloy is characterized by its typical large shape hysteresis loop (high coercivity), which always requires an excellent controller to obtain the desired induction waveform. All measurements are well performed, but as can be observed at $5 \mathrm{kHz}$, there are saw-tooth variations around the remanence area of the hysteresis loop. This phenomenon is due to the bandwidth 
limit of the power amplifier. Since the cut-off frequency is $100 \mathrm{kHz}$, harmonics above 20 are distorted, and therefore the induction obtained is slightly distorted but still with acceptable quality.

More complicated measurements, which are essential to quantify the losses increment produced by the switched-mode power supply in magnetic materials, are reported in Fig. 11. A PWM waveform (a) and an asymmetrical triangle waveform (b) are respectively imposed on the flux density. In the case of Fig. 11.b, a symmetrical triangle of $1.5 \mathrm{~T}$, which is not shown, must be added at the beginning of the signal to calibrate the magnetization state of the material. The calibration of the magnetization state is important, because the voltage sensor can only determine the difference between the maximum and minimum value of flux density, but not the actual absolute value of flux density. The material must also be demagnetized before the measurement. At $50 \mathrm{~Hz}$, the algorithm converges after approximately 5 iterations. The waveform with DC offset is not tested in this study. This type of measurements requires additional equipment (fluxmeter, adapted power amplifier,...) and investigations. However, our controller promises to work well.

Summary of the controller performance is given in Table II. This controller is adaptable for all tested electrical steels from conventional to unconventional, with various waveform ranging from simple to very complex, and with induction up to $95 \%$ of the saturation. On the other hand, the dynamic measurement quality is specified by the ADC sampling of the acquisition cards and especially by the bandwidth of the power amplifier. With a more advantageous power amplifier, the magnetizing frequency promises to rise even higher.

\section{CONCLUSION}

This paper was about a novel digital flux density controller. Its principle was described, together with the implementation in a soft magnetic material measurement bench. The idea of separating the controlled signal into two terms, including the amplitude and the normalized waveform, allows the system to have great stability and high accuracy in conformity with the international standard. Due to the non-linearity of materials, the controller convergence speed is dependent on measurement conditions such as type of tester, material, magnetizing frequency, and required flux density. However, in any case, it is still more relevant and robust than that reported in the other publications. Without any extra parameter or complicated identification procedure, this controller is highly adaptable to any 1D magnetic measurements. Application in 2D and 3D systems shall be carried out in the subsequent studies.

\section{REFERENCES}

[1] S. Tumanski, Handbook of Magnetic Measurements. CRC Press, Apr 2016.

[2] F. Fiorillo, Measurement and characterization of magnetic materials. North-Holland, 2004.

[3] I. E. Commission, "Magnetic materials - Part 2: Methods of measurement of magnetic properties of electrical steel strip and sheet by means of an Epstein frame," 1996.

[4] T. Chevalier, A. Kedous-Lebouc, B. Cornut, and C. Cester, "A new dynamic hysteresis model for electrical steel sheet," Physica B: Condensed Matter, vol. 275, no. 1, pp. 197-201, Jan. 2000.
[5] G. Bramerdorfer and D. Andessner, "Accurate and Easy-to-Obtain Iron Loss Model for Electric Machine Design," IEEE Transactions on Industrial Electronics, vol. 64, no. 3, pp. 2530-2537, Mar. 2017.

[6] S. E. Zirka, Y. I. Moroz, P. Marketos, and A. J. Moses, "Viscosity-based magnetodynamic model of soft magnetic materials," IEEE Transactions on Magnetics, vol. 42, no. 9, pp. 2121-2132, Sep. 2006.

[7] E. Dlala, A. Belahcen, K. A. Fonteyn, and M. Belkasim, "Improving Loss Properties of the Mayergoyz Vector Hysteresis Model," IEEE Transactions on Magnetics, vol. 46, no. 3, pp. 918-924, Mar. 2010.

[8] D. Gerada, A. Mebarki, N. L. Brown, C. Gerada, A. Cavagnino, and A. Boglietti, "High-Speed Electrical Machines: Technologies, Trends, and Developments," IEEE Transactions on Industrial Electronics, vol. 61, no. 6, pp. 2946-2959, Jun. 2014.

[9] A. Krings, A. Boglietti, A. Cavagnino, and S. Sprague, "Soft Magnetic Material Status and Trends in Electric Machines," IEEE Transactions on Industrial Electronics, vol. 64, no. 3, pp. 2405-2414, Mar. 2017.

[10] A. Krings, "Iron losses in electrical machines-influence of material properties, manufacturing processes, and inverter operation," Ph.D. dissertation, KTH Royal Institute of Technology, 2014.

[11] A. Boglietti, A. Cavagnino, D. M. Ionel, M. Popescu, D. A. Staton, and S. Vaschetto, "A General Model to Predict the Iron Losses in PWM Inverter-Fed Induction Motors," IEEE Transactions on Industry Applications, vol. 46, no. 5, pp. 1882-1890, Sep. 2010.

[12] K. Matsubara, N. Takahashi, K. Fujiwara, T. Nakata, M. Nakano, and $\mathrm{H}$. Aoki, "Acceleration technique of waveform control for single sheet tester," IEEE Transactions on Magnetics, vol. 31, no. 6, pp. 3400-3402, Nov. 1995.

[13] S. A. Spornic, A. Kedous-Lebouc, and B. Cornut, "Numerical waveform control for rotational single sheet testers," Le Journal de Physique IV, vol. 08, no. PR2, pp. Pr2-741-Pr2-744, Jun. 1998.

[14] D. Makaveev, J. Maes, and J. Melkebeek, "Waveform control algorithm for rotational single sheet testers using system identification techniques," Journal of Applied Physics, vol. 87, no. 9, pp. 5983-5985, Apr. 2000.

[15] N. Derebasi, R. Rygal, A. J. Moses, and D. Fox, "A novel system for rapid measurement of high-frequency magnetic properties of toroidal cores of different sizes," Journal of Magnetism and Magnetic Materials, vol. 215-216, pp. 684-686, Jun. 2000.

[16] S. Zurek, P. Marketos, T. Meydan, and A. J. Moses, "Use of novel adaptive digital feedback for magnetic measurements under controlled magnetizing conditions," IEEE Transactions on Magnetics, vol. 41, no. 11, pp. 4242-4249, Nov. 2005.

[17] S. Zurek, "Practical implementation of universal digital feedback for characterisation of soft magnetic materials under controlled AC waveforms," in Symposium of Magnetic Measurements \& Modeling, Poland, 2016.

[18] P. Anderson, "Measurement of the stress sensitivity of magnetostriction in electrical steels under distorted waveform conditions," Journal of Magnetism and Magnetic Materials, vol. 320, no. 20, pp. e583-e588, Oct. 2008.

[19] Z. Polik and M. Kuczmann, "Measuring and control the hysteresis loop by using analog and digital integrators," J. Optoelectron. Adv. Mater, vol. 10, no. 7, pp. 1861-1865, 2008.

[20] P. Marketos, J. P. Hall, and S. E. Zirka, "Power Loss Measurement and Prediction of Soft Magnetic Powder Composites Magnetized Under Sinusoidal and Nonsinusoidal Excitation," IEEE Transactions on Magnetics, vol. 44, no. 11, pp. 3847-3850, Nov. 2008.

[21] S. White, T. W. Krause, and L. Clapham, "A Multichannel Magnetic Flux Controller for Periodic Magnetizing Conditions," IEEE Transactions on Instrumentation and Measurement, vol. 61, no. 7, pp. 1896-1907, Jul. 2012.

[22] O. Stupakov and P. Svec, "[Three Parameter Feedback Control of Amorphous Ribbon Magnetization," Journal of Electrical Engineering, vol. 64, no. 3, pp. 166-172, May. 2013.

[23] M. G. Blundell, K. J. Overshott, and C. D. Graham Jr, "A new method of measuring power loss of magnetic materials under sinusoidal flux conditions," Journal of Magnetism and Magnetic Materials, vol. 19, no. 1-3, pp. 243-244, 1980.

[24] F. Fiorillo and A. Novikov, "An improved approach to power losses in magnetic laminations under nonsinusoidal induction waveform," IEEE Transactions on Magnetics, vol. 26, no. 5, pp. 2904-2910, 1990.

[25] J. C. Doyle, B. A. Francis, and A. R. Tannenbaum, Feedback control theory. Courier Corporation, 2013.

[26] M. Baker, Demystifying mixed signal test methods. Elsevier, 2003. 